\title{
A New Healthcare Context Information: The Social Context
}

\author{
Isra'a Ahmed Zriqat \\ Department of Computer Science \\ Applied Science Private University \\ Amman, Jordan
}

\author{
Ahmad Mousa Altamimi \\ Department of Computer Science \\ Applied Science Private University \\ Amman, Jordan
}

\begin{abstract}
During the treatment process, medical institutes collect context information about their patients and store it in their healthcare systems. The collected information describes the measurable, risk, or medication information and used to improve the performance of the institutes healthcare systems by allowing diverse knowledge about patients. Being said that some other information is needed as they influence patients' life style such as education and income as the high level of education or income reflected positively to the patient's life, and probably resulting in reducing likelihood disease or incidence of infectious diseases. In this paper, a new type of healthcare context information (Social Context) is proposed to address this need. It can be divided into four main categories: related people, behavior, income and education of the patient. We believe that the new proposed context information should be considered in the designing process of the context-aware medical informatics systems beside to the well-known context information.
\end{abstract}

Keywords-Context information; social context; healthcare; medical information

\section{INTRODUCTION}

The concept of Context is treated differently in the literature, a review discloses large number of definitions. However, authors of [1] proposed the most refer definition that is "any information that can be used to characterize the situation of entities (i.e., whether a person, place, or object)". The "situation" here refers to a description of the states of relevant entities. According to this definition, different types of information are needed to be gathered and analyzed to characterize an entity's context such as: Identity, Location, Time, Status, Physical context, Emotional context, etc. [1]-[3]. In fact, all the context information cannot be listed here due to their diversity types and numerous number. Therefore, in this work, we will describe only the context information relevant to the health system.

Here, the well-known context information (e.g., Personal Information, location and time) [4]-[13] are considered along with our new context aspect (e.g., Social Context). Together, they describe the patient's social context and stores data about his/her surrounding peoples. For example, tracking the location of patients assists caregivers, especially in emergencies. This will off course improve the performance of healthcare monitoring system.

In fact, there are many cases where tracking the patients place or time is needed. For example, elderly patients vulnerable to fall more than others due to fall risks. A hardphysical exercise will influent the medical decision as the blood pressure will be high. In this regard, technology such as Real Time Locating System (RTLS) could be used to accurately detecting falls, and can measure many parameters (e.g., Body temperature, respiration rate, heart rate, blood pressure, and ECG) of patient continuously, and then transmitted wirelessly [10], [11]. This results in improving patient care and reducing associated health care costs [12], [13].

In this paper, a new context aspect (e.g., Social Context) is proposed that includes information influence the patient's health such as: the patient's social information and related people. The proposed factor can be categorized into four main categories: related people of the patients, behavior of the patients, income and the education of the patients. Such data should be considered in the designing of medical contextaware systems as they influence the patient's life style. For example, the high level of education or income reflected positively to the patient's life, and probably resulting in reducing likelihood disease or incidence of infectious diseases. Furthermore, some patients may have communicable diseases that may spread and infect all who have contact them.

The structure of this paper is as follow. Section II presents the related work. The research methodology of our work is then given in Section III. The proposed Social Context Information is then discussed in Section IV. Finally, the conclusion and the future work are offered in Section V.

\section{RELATED WORK}

Context information has been extensively considered in the literature. Patient's personal information, location, and time context for instance have reviewed in [7]-[11]. Because our work is related to healthcare, and because of the lack of simplicity, we will consider the Context Information that is used for describing Heart Diseases as illustrated in Fig. 1. In fact, context information is primarily divided into two main categories: Personal and location and time context.

\section{A. Personal Context Information}

There are different types of patient's personal information to be considered such as patient's vital signs, the associated risk factors with disease, medical symptoms, and current prescribed medications. Next, we give brief description for each type. 


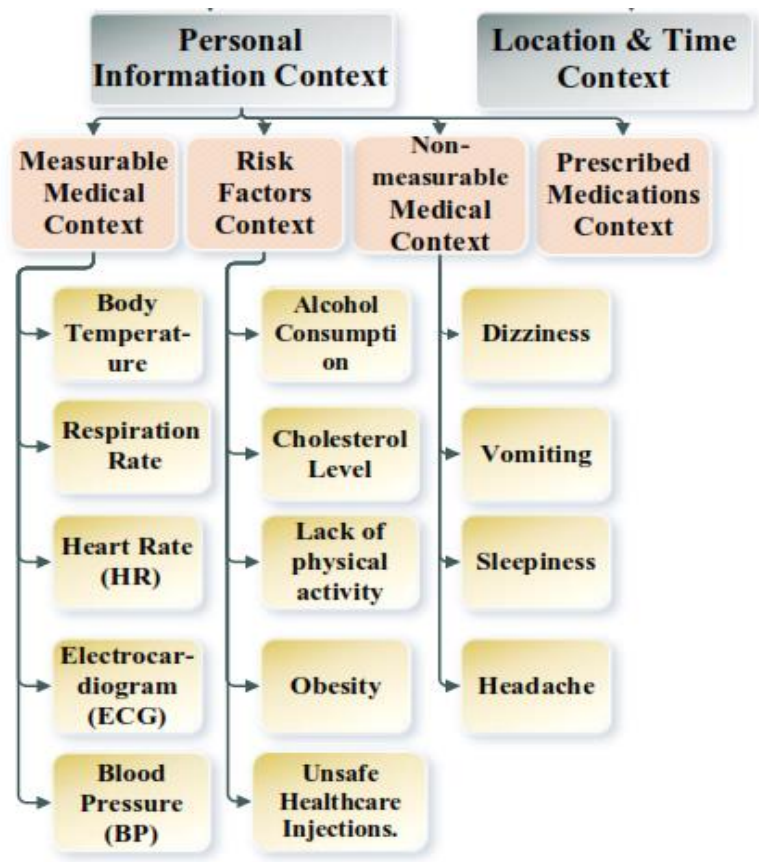

Fig. 1. Types of heart diseases' context information.

1) Measurable Medical Context Information: includes vital signs that can be measured and continually monitored such as: body temperature, heart rate, blood pressure, respiration rate, etc. [4], [5], [8].

2) Risk Factors Context: covers the health factors that changed infrequently [4], [7]. According to the World Health Organization, these factors are countless, and a set of these risk factors can be combined leading cause of a specific disease. For instance, there are seven risk factors associated with cancer disease (e.g., tobacco, alcohol, air pollution, low fruit and vegetable intake, physical inactivity, unsafe sex, and unsafe healthcare injections).

3) Non-measurable medical context information: which are symptoms that are difficult to be measured such as: headache, vomiting, sleepiness, and dizziness. Despite their measuring difficulties, they should be considered along with the measurable context in the healthcare context [9], [14].

4) Medications context information: provide the current medications given for a patient as it affects the readings of patient's vital signs [9], [14], [15]. Thus, healthcare givers evaluate the patient's response to take the appropriate medical decisions [9].

\section{B. Location and Time Context Information}

It is considering the location of patients to improve the performance of healthcare monitoring system. There are many cases where tracking the patients place is needed. For example, elderly patients vulnerable to fall more than others due to fall risks. In this regard, technology such as Real Time Locating System, an embedded microcontroller is connected to a set of medical sensors and a wireless channel to measure and transfer the patient's sign continuously [10], [11], can be employed to accurately detecting falls, which improves healthcare services and reduces the associated costs [12], [13].

\section{METHODOLOGY}

There are many types of context information that have been considered in the literature [4]-[13]. These types can be considered together for describing the patient's context, and designing the medical informatics systems. Such systems should contain all the relevant patient's information to deliver proper service. Beside the well-known contexts information, other context information such as the social aspect context should be considered as it is describing the patient's social context and stores data about his/her surrounding peoples. The proposed aspect consists of data about behavior, income, education of the patient, and data about his/her related people that influences the patients' health.

\section{SOCIAL CONTEXT INFORMATION}

Social context relates to the social and community that surrounded patients. Those patients may have communicable diseases that may spread to infect all who have relationship with them. In addition, aspects related to social functioning are considered as highly relevant by patients and their family members. Regardless of these aspects, pharmacotherapy and any doubts about their illness, caregivers, home nursing, behavior, and other social aspects present private characteristics that need to be secured from misusing or unauthorized actions [16].

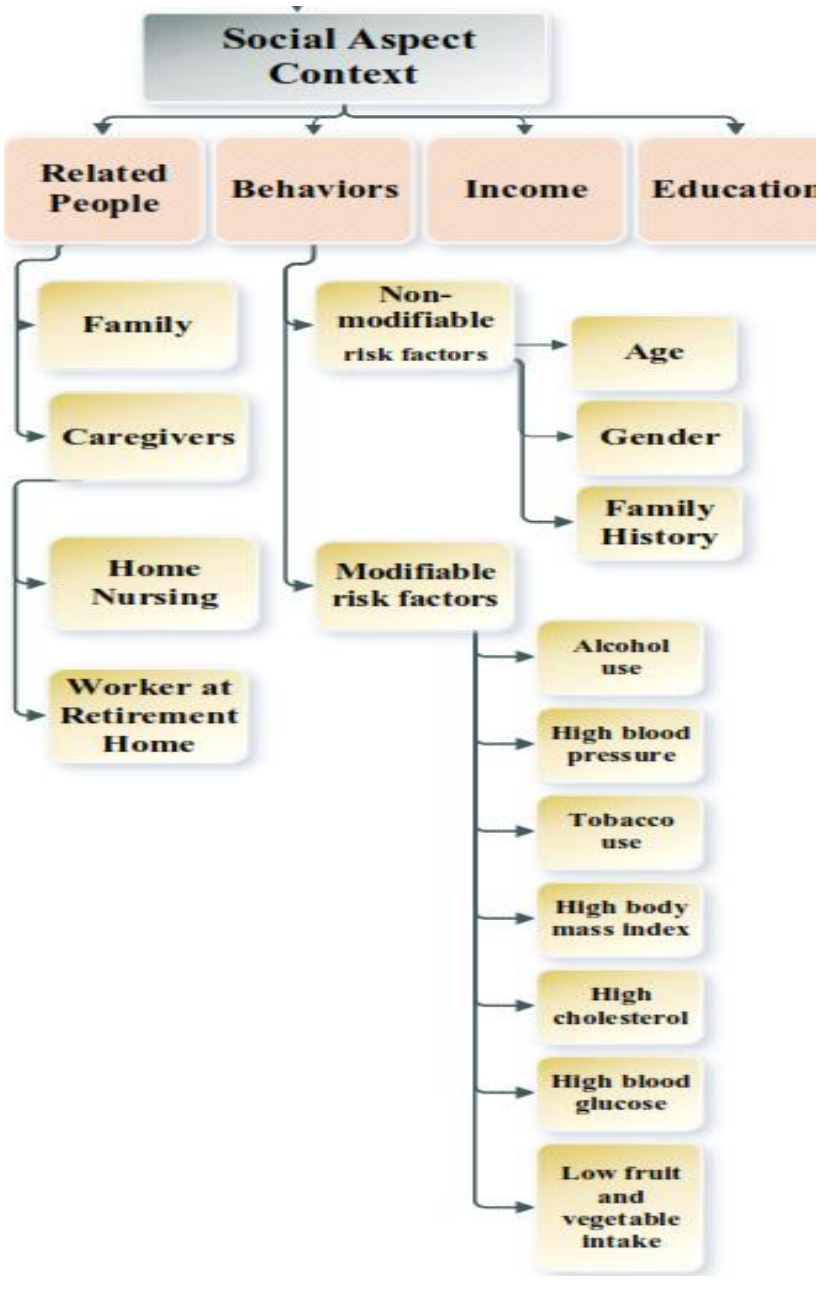

Fig. 2. Proposed social information context. 
The proposed social aspect context could include: related people of the patients, behavior of the patients, income and education as illustrated in Fig. 2. Next, a brief description for each category is given.

\section{A. Related People}

Related peoples refer to those who have a relationship with the patient, such as family members, nurses, and other caregivers. This category could be:

- Family members: refer to individuals who have direct connect with their patients. Since those people live with them at the same place, their information should be kept secret if it is stored in the eHealth system.

- Caregivers: refer to anyone who take care of patients or their work require to deal with patients. Caregivers could be: home nursing who live at home and provide round-the-clock care or long-term medical treatment [17]. Or, workers at retirement home who work at place for the individuals who are still able to care for themselves independently, but have difficulty managing an entire house [17]. We iterate that the information of such related people should be kept secure (if stored in the eHealth systems) as patient may suffer from several types of infectious diseases and could affect the caregiver or those who live with them at the same house.

\section{B. Behavior}

According to World health federation, it can be divided into Modifiable and Non-modifiable risk factors. The Modifiable health risk factors that associated with heart disease could contain alcohol use, high blood glucose, low fruit and vegetable intake, tobacco use, high body mass index, high cholesterol, high blood pressure, and physical inactivity. As long these risk behaviors have a negative effect on individual health and account for $61 \%$ of heart disease deaths [17]. All these risk behaviors considered as private information that should not be exposed to unauthorized people. In the other hand, the modifiable risk factors refer to the factors that cannot be changed such as age, gender and family history [18]. Whereas, the heart disease becomes increasingly common with advancing age, since the heart undergoes subtle physiological changes, as a person gets older. Also, your gender is significant, as a female is at less risk of heart disease than a male. As well as, the risk will increase if a first-degree relative suffers heart diseases, as the family's history could reveal your possibilities. Family's history of heart disease could reveal your possibility [18].

\section{Income and Education}

Many comparative studies found that income primarily influences health [19], [20]. Those studies showed that high income allowing the individuals for buying better quality material goods such as food and shelter, and allowing them to access health services and leisure activities, which make their bodies healthy that in turn reduces the likelihood of disease [19], [20]. On the other hand, there are specific interpretations explain that the education has a large influence on health [19], [21]. Patients with high level education could improve mutual comprehension between them and their physicians, resulting in improving quality of care [20]. As well as, several studies found that correlation between high level education and the personal management of several diseases like spreading of sexually transmitted diseases.

\section{CONCLUSION AND FUTURE WORK}

In this work, we propose new context that includes the social context patients' information. Such information influences patients' health and life style. For example, the high income provides decent life and reflected positively on the patient's health, resulting in reduces the likelihood of disease. In addition, patients may have infectious diseases, which will be spread and infect all who have relationship with patients. All these data should be considered in the designing of healthcare systems and should be kept secured and protected from unauthorized accessing. As a future work, we are planning to develop a context-aware access control model for eHealth systems that will be built specifically for healthcare systems.

\section{ACKNOWLEDGMENT}

The authors are grateful to the Applied Science Private University, Amman-Jordan, for the full financial support granted to cover the publication fee of this research article.

\section{REFERENCES}

[1] Dey, A.K., Abowd, G.D., and Salber, D.: 'A conceptual framework and a toolkit for supporting the rapid prototyping of context-aware applications', Human-computer interaction, 2001, 16, (2), pp. 97-166.

[2] A. Dey: "Context-aware computing: The CyberDesk project", Proceedings of the AAAI 1998 Spring Symposium on Intelligent Environments, AAAI Press, Menlo Park, CA, 1998, pp. 51-54.

[3] Ch. Bornträger: "Contextual Influence on the Usability of Different Media Types”, Diploma Thesis, Technische Universität Ilmenau, Ilmenau, Jan. 2003.

[4] Roy, N., Gu, T., and Das, S.K.: 'Supporting pervasive computing applications with active context fusion and semantic context delivery', Pervasive and Mobile Computing, 2010, 6, (1), pp. 21-42.

[5] Copetti, A., Loques, O., Leite, J.C., Barbosa, T.P., and da Nobrega, A.C.: 'Intelligent context-aware monitoring of hypertensive patients', in Editor: 'Book Intelligent context-aware monitoring of hypertensive patients' (IEEE, 2009, edn.), pp. 1-6.

[6] Smith, S.F., and Duell, D.: 'Clinical nursing skills: Nursing process model, basic to advanced skills' (McGraw-Hill/Appleton \& Lange, 1992).

[7] Organization, W.H.: 'Global health risks: mortality and burden of disease attributable to selected major risks' (World Health Organization, 2009. 2009).

[8] Gardner, R.M., and Shabot, M.M.: 'Patient-monitoring systems': 'Biomedical Informatics' (Springer, 2006), pp. 585-625.

[9] Al-Bashayreh, M.G., Hashim, N.L., and Khorma, O.T.: 'Context-Aware Mobile Patient Monitoring Frameworks: A Systematic Review and Research Agenda', JSW, 2013, 8, (7), pp. 1604-1612.

[10] Shaikh, R.A.: 'Real time health monitoring system of remote patient using ARM7', Control and Automation (IJICA) ISSN, 2012, pp. 22311890 .

[11] Al-Aubidy, K.M., Derbas, A.M., and Al-Mutairi, A.W.: 'Real-time patient health monitoring and alarming using wireless-sensor-network', in Editor: 'Book Real-time patient health monitoring and alarming using wireless-sensor-network' (IEEE, 2016,), pp. 416-423.

[12] Bowen, M.E., Craighead, J., Wingrave, C.A., and Kearns, W.D.: 'RealTime Locating Systems (RTLS) to improve fall detection', Gerontechnology, 2010, 9, (4), pp. 464-471. 
[13] Boulos, M.N.K., and Berry, G.: 'Real-time locating systems (RTLS) in healthcare: a condensed primer', International journal of health geographics, 2012, 11, (1), pp. 25.

[14] Koutkias, V.G., Chouvarda, I., Triantafyllidis, A., Malousi, A., Giaglis, G.D., and Maglaveras, N.: 'A personalized framework for medication treatment management in chronic care', IEEE transactions on information technology in biomedicine, 2010, 14, (2), pp. 464-472.

[15] Mohomed, I., Misra, A., Ebling, M., and Jerome, W.: 'Harmoni: Context-aware filtering of sensor data for continuous remote health monitoring', in Editor: 'Book Harmoni: Context-aware filtering of sensor data for continuous remote health monitoring' (IEEE, 2008, edn.), pp. 248-251

[16] Organization, W.H.: 'The economics of social determinants of health and health inequalities: a resource book' (World Health Organization, 2013. 2013).
[17] Alzhimer's Association®. Avaliable $\mathrm{http} / / / \mathrm{www}$. alz.org/care/alzheimers-dementia-residentialfacilities.asp\#ixzz4L0xPe3kH

[18] World health federation. http://www.world-heartfederation.org/press/fact-sheets/cardiovascular-disease-risk-factors/. Accesed on 7 June 2017.

[19] Organization, W.H.: 'A conceptual framework for action on the social determinants of health', 2010.

[20] Lepage, B., Schieber, A.-C., and Lamy, S.: 'Social determinants of cardiovascular diseases', Public Health Reviews, 2011, 33, (2).

[21] Woolf, S.H., and Braveman, P.: 'Where health disparities begin: the role of social and economic determinants - and why current policies may make matters worse', Health affairs, 2011, 30, (10), pp. 1852-1859. 